REVISTA EVIDENCIAÇÃO CONTÁBIL \& FINANÇAS

João Pessoa, v. 7, n. 1, p. 78-93, jan./abr. 2019. ISSN 2318-1001

DOI: 10.22478/ufpb.2318-1001.0v0n0.37439

Disponível em: http://periodicos.ufpb.br/ojs2/index.php/recfin

\title{
ANÁLISE DA INFLUÊNCIA DO CENÁRIO ECONÔMICO INTERNO E EXTERNO NA PRÁTICA DE INCOME SMOOTHING EM BANCOS BRASILEIROS ${ }^{1}$
}

\section{ANALYSIS OF THE INFLUENCE OF THE INTERNAL AND EXTERNAL ECONOMIC SCENARIO IN THE PRACTICE OF INCOME SMOOTHING IN THE BRAZILIAN BANKS}

\author{
Wesley Paulo dos Santos ${ }^{2}$ \\ Faculdade Santa Helena (FSH) \\ wesley paulo@live.com \\ https://orcid.org/0000-0002-2166-0919 \\ Raimundo Nonato Rodrigues \\ Doutor em Ciências Contábeis/USP \\ Universidade Federal de Pernambuco(UFPE) \\ rnrdg@uol.com.br \\ https://orcid.org/0000-0003-0505-4369
}

Mestre em Ciências Contábeis pelo Programa de Pós-Graduação em Ciências Contábeis UFPE

\section{Luiz Carlos Marques dos Anjos}

Doutor em Ciências Contábeis pelo Programa Multi-institucional e Interregional de Pós-Graduação em Ciências Contábeis UnB/UFPB/UFRN

Universidade Federal de Pernambuco (UFPE)

luiz.cmanjos@ufpe.br

https://orcid.org/0000-0002-7375-540X

\section{Márcia Ferreira Neves Tavares}

Doutora em Ciências Contábeis pela UnB/UFPB/UFRN

Universidade Federal de Pernambuco (UFPE)

marcia@ferreiraauditores.com.br

https://orcid.org/0000-0001-9229-797X

\section{RESUMO}

Objetivo: Avaliar se o cenário econômico interno ou externo representado pelo produto interno bruto do Brasil e de outras economias exerceu alguma influência para os bancos que compõe o sistema financeiro nacional a praticarem income smoothing.

Fundamentos: Sobre a suavização de resultado, Sood (2012) e Dolar (2016) demonstraram que em cenários econômicos internos recessivos, os bancos norte-americanos tenderam a não praticar income

\footnotetext{
${ }^{1}$ Artigo recebido em: 05/12/2017. Revisado por pares em: 27/04/2018. Reformulado em: 21/05/2018. Recomendado para publicação, após a terceira rodada, em: 24/05/2018 por Luiz Felipe de Araújo Pontes Girão (Editor Geral). Publicado em: 21/11/2018. Organização responsável pelo periódico: UFPB

2 Endereço: Av. Caxangá, Madalena, Recife - PE, 50610-120
} 
smoothing. Dessa forma, o problema deste estudo é verificar se os bancos que compõe o sistema financeiro brasileiro praticaram income smoothing entre o período de 2011 até o segundo semestre de 2017, período esse, de desaceleração da economia brasileira e que culminou em uma recessão econômica.

Método: Adotou-se a regressão logit em painel com efeitos aleatórios. A suavização de resultado foi medida pelo índice de Eckel (1981) e sua relação foi testada com os cenários econômicos do Brasil e de diferentes economias globais.

Resultados: Este estudo identificou que os PIBs de outras economias foram significantes para a prática de income smoothing realizada pelos bancos brasileiros. Os resultados foram significativos para a evolução econômica do EUA e do G7.

Contribuições: Este estudo contribui para a literatura por identificar que cenários econômicos de outras economias globais podem influenciar na prática de suavização de resultado dos bancos de uma economia local.

Palavras-chave: Income Smoothing, Cenário Econômico Interno, Cenário Econômico Externo.

\section{ABSTRACT}

Objective: To evaluate whether the internal or external economic scenario represented by the gross domestic product of Brazil and other economies, exerted some influence for the banks that make up the national financial system to practice income smoothing.

Fundamentals: On smoothing Sood (2012) and Dolar (2016) have demonstrated that in recessive domestic economic scenarios, US banks tended not to practice income smoothing. Thus, the potential problem of this study is to verify if the banks that compose the Brazilian financial system practiced income smoothing between the 2011 until the second half of 2017, a period of slowdown in the Brazilian economy that culminated in an economic recession.

Method: The logit regression in panel with fixed effects was adopted. The smoothing of results was measured by the Eckel index (1981) and its relation was tested statistically with the economic scenarios of Brazil and different global economies.

Results: This study identified the GDPs of other economies as significant for the income smoothing practice of Brazilian banks. The results were important for the economic evolution of the US and the G7.

Contributions: This study contributes to the literature by identifying which economic scenarios from other global economies may influence the practice of smoothing the outcome of banks in a local economy.

Key words: Income Smoothing, Internal Economic Scenario, External Economic Scenario.

\section{INTRODUÇÃO}

Os bancos, assim como outras empresas, gerenciam seus resultados de maneira artificial, tendo em vista algum interesse específico, em que essa prática ficou conhecida como income smoothing. Beidleman (1973) define income smoothing como um amortecimento intencional das flutuações do lucro a um nível que atualmente é considerado como aceitável para a empresa. Assim, o income smoothing ao mesmo tempo em que antecipa futuros resultados previstos pela empresa, também distorce o seu resultado atual.

Sobre a suavização de resultado, é vasta a literatura que trata sobre sua prática em relação ao setor bancário, que em linhas gerais se dividem entre estudar os mecanismos utilizados para a prática de income smoothing e aqueles que possuem como objetivo determinar sua motivação. Sobre o 
mecanismo utilizado, Bath (1996), Ahmed, Takeda e Thomas (1999) e Kilic, Lobo, Ranasinghe e Sivaramakrishnan (2013) identificaram que accruals discricionários, na forma de provisões para perda de crédito, são os mecanismos centrais utilizados pelos bancos para a suavização do resultado.

Em relação a motivação, vários fatores já foram considerados, como a regulação contábil imposta aos bancos. Sobre isso, Tucker e Zarowin (2006) e Cahan, Liu e Sun (2008) sugerem que empresas que atuam em países com baixa proteção aos investidores tendem a suavizar mais seus resultados, de maneira oportunista, e com isso, prejudicar a qualidade da informação contábil.

Outra motivação encontrada para a suavização de resultado diz respeito ao cenário econômico enfrentado pela empresa. DeFound e Park (1997), Healy e Walen (1999), Liu e Ryan (2006), Bushman e Williams (2012) e Dolar (2016) identificaram como principal fator para o gerenciamento de resultado, as expectativas das empresas sobre futuros cenários econômicos, considerando o Produto Interno Bruto (PIB) como variável explicativa. Dessa maneira, os resultados encontrados por esses estudos informam que os bancos possuem estímulos para suavizar resultados em momentos de crescimento e estabilidade econômica de maneira a diminuir lucro e manter caixa para que os ativos possam ser utilizados em momentos de baixo crescimento econômicos ou de recessão.

No Brasil, o estudo de Goulart (2007) analisou tanto a motivação quanto o mecanismo utilizado pelos 49 bancos maiores bancos brasileiros nos períodos entre 2002 e 2006, em função do cenário interno brasileiro e concluiu que os bancos brasileiros utilizaram a provisão para perda sob crédito como principal meio para o gerenciamento de resultado. Outro estudo realizado com bancos brasileiros foi o de Dantas, Medeiros, Galdi e Costa (2013). Eles analisaram os bancos entre o período de 2002 a 2010 e os resultados encontrados por esse estudo, concordam com o que foi identificado por Goulart (2007), de que o principal mecanismo utilizado pelos bancos para a prática de income smoothing foi a provisão para perdas.

Apesar de já existirem trabalhos que possuíram como objetivo a suavização de resultado feita pelos bancos brasileiros, eles se concentraram na forma e no cenário interno como justificativa para as pesquisas. Assim, este estudo se justifica por levar em consideração o cenário econômico interno além dos períodos já estudados. Uma vez que Goulart (2007) e Dantas et al. (2013) consideraram, respectivamente, o período de 2002 a 2006 e de 2002 a 2011, este trabalho se concentrou no período que compreendem os anos de 2011 a 2017, período este de recessão para a economia brasileira. Este trabalho, também, se diferencia ao considerar possíveis influências que a evolução de outras economias pode acarretar aos bancos brasileiros, uma vez que essas empresas podem investir ou possuir sedes ou filiais em outros países além do Brasil.

Dessa forma, este estudo tem como objetivo analisar se o que exerceu alguma influência na prática de gerenciamento de resultado realizada pelos bancos comerciais brasileiros foi a expectativa interna de agentes do mercado em relação ao cenário econômico seguinte, ou se foi em resposta ao cenário econômico presente, seja interno ou externo. O período considerado compreende o primeiro trimestre de 2011 ao segundo trimestre de 2017. A amostra considerada foram os 40 bancos que se mantiveram presentes na lista dos 50 maiores bancos brasileiros, na categoria b1, nos períodos de 2011 a 2017.

Tal investigação é necessária para identificar se os maiores bancos brasileiros antecipam seus resultados na forma de income smoothing, por levar em consideração as expectativas dos principais operadores do mercado financeiro ou se a suavização de resultado é apenas alguma resposta a algum cenário presente, seja interno ou externo. Para isso buscou identificar, inspirado pelos trabalhos de Galai e Winer (2003), Liu e Ryan (2006), Sood (2012) e Dolar (2016), se os principais bancos brasileiros não possuíam estímulos a suavizarem seus resultados tendo em vista o cenário econômico recessivo apresentado pelo Brasil entre 2015 e 2016 ou, ainda, se algum cenário econômico externo influenciou a suavização de resultado. Os resultados encontrados apontaram uma maior prática de income smoothing no segundo e quarto trimestre de cada ano investigado. 


\section{REVISÃO DE LITERATURA}

\section{1- Suavização de resultado e cenário econômico}

O income smoohting é um ato cometido pelos gestores da empresa com o objetivo de maximizar a sua própria utilidade esperada, mesmo que não reflita o interesse dos acionistas (Lambert, 1984). E por definição, income smoothing é um amortecimento intencional das flutuações do lucro a um nível que seja considerado como aceitável para a empresa (Beidleman, 1973). Em pesquisa realizada por Graham, Harvey e Rajgopal (2005) com os CFOs, 96,9\% deles indicaram que partem da premissa de que a empresa gerencia seus ganhos, indicando que preferem um caminho de ganhos mais suaves.

DeFound e Park (1997) e Healy e Walen (1999) expõem que a causa do gerenciamento de resultado é o julgamento que é feito pela gestão da empresa sobre futuros cenários econômicos. No entanto, Albrechta e Richarson (1990) ao considerar as empresas da economia dos EUA, relatam que a suavização de resultado e a motivação para tal ato variam de acordo com o setor econômico que a empresa está inserida. Tucker e Zarowin (2006) ao analisar várias empresas entre o período de 1993 a 2000, verificaram que a suavização de resultado fornece um aumento de "informatividade" dos ganhos correntes ao incorporar informações sobre cenários econômicos futuros em suas demonstrações. Já Liu e Ryan (2006) fornecem como causa para a prática de income smoohting, a manutenção de caixa que este mecanismo possibilita para as empresas.

Diversos estudos já analisaram a relação entre o caixa das empresas e cenário econômico. Richardson (2006), Gulati, Nohria e Wohlgezogen (2010) e Nason e Patel (2016) apontaram que em períodos que antecedem uma recessão econômica, as empresas tendem a formar caixa para que durante a recessão, o caixa possa ser utilizado. Uma vez que a palavra "recessão" é um termo adotado pela economia, se faz necessário uma definição. Blanchard (2007) define recessão como o déficit anual do Produto Interno Bruto (PIB) de uma nação ou o resultado negativo do PIB em dois trimestres consecutivos (recessão técnica).

Copeland (1968) elencou as características para o que chamou de uma perfeita utilização do income smoothing, a saber: a) Quando utilizado não deve comprometer nenhuma ação futura da empresa; b) Deve ser considerado dentro do domínio das normas de contabilidade; c) Deve levar em consideração, de forma material, os desvios relativos as diferenças dos lucros de ano para ano; d) Não deve exigir uma transação real ou externa, mas apenas uma reclassificação de valores das contas internas; e) Deve ser considerado isoladamente ou de forma conjunta, na ocorrência de outras práticas ao longo de períodos de tempo consecutivos.

Gao e Zhang (2015) ao estudar 2.022 firmas, para o período de 1993 a 2010, considerou que a suavização de resultado pode ser realizada tanto de maneira informativa quanto de modo oportunista pelos responsáveis pela gestão da empresa. Para Jayaraman (2008), se realizado de maneira oportunista, além de distorcer o conteúdo informativo contemporâneo dos ganhos da empresa, a empresa e seus gestores não estarão informando as suas previsões sobre os cenários econômicos futuros.

Dessa forma, se bem empregada a suavização de resultado, segundo Chaney e Lewis (1995), isso levará a uma relação ainda questionável, pois por um lado os ganhos não representarão com precisão os ganhos econômicos em cada ponto no tempo, por outro lado, a suavização levará a empresa a revelar mais informações privadas sobre os possíveis lucros e fluxos de caixa decorrentes de um cenário econômico futuro.

\section{2- Income smoothing no setor bancário}

De acordo com Kanagaretnam, Lobo e Yang (2004) são vários os motivos para a prática de income smoothing pelos bancos; onde alguns dos motivos são: 1- A gestão de capital realizada pelos 
gestores bancários; 2- A busca do nivelamento do lucro. 3-Sinalização de estabilidade para o mercado.

Corroborando com isso, Côté (2016) relaciona a busca por nivelamento do lucro com a sinalização de estabilidade apresentada pelos bancos ao mercado. Ao analisar os bancos canadenses dos anos de 1996 a 2000, a luz da Teoria da Legitimidade, Côté (2016) verificou que apesar do cenário econômico recessivo ou de baixo crescimento, os bancos canadenses continuavam a apresentar lucros maiores, sendo que a parcela do lucro ficava represada na forma de income smoohting. Esta representou uma situação em que a prática de income smoohting pouco informava sobre o futuro cenário econômico previsto para a entidade. O que levou Côté (2016) a concluir que, uma das formas que os bancos possuem de passar confiança para o mercado, é aumentando os seus lucros; porém apesar desse estimulo, os bancos, ainda que tenham capacidade, só aumentarão os seus lucros até um nível que os gestores entendam que ainda seja considerado como aceitável para a maioria dos stackeholders, caso contrário, poderá colocar em risco a legitimidade social da instituição.

Liu e Ryan (2006) ao analisar as holdings bancárias, que estavam sob a regulação da SEC, para os anos de 1991 a 2000 argumentaram que os bancos têm incentivos para diminuírem seus lucros em períodos bons, para permitir o aumento de seus resultados nos maus momentos subsequentes, por meio da liberação de "reservas ocultas" constituída pela prática do income smoothing. De forma anterior a Liu e Ryan (2006), Galai e Winer (2003) apontam como causa desse comportamento, por parte do setor bancário, a necessidade que há desse setor, em transmitir confiança para o mercado em momentos econômicos desfavoráveis, satisfazendo, assim, as previsões dos analistas de mercado ou exigências de capital.

Kilic et al. (2013) ao estudar o mecanismo que os bancos norte-americanos utilizaram para suavizar seus resultados nos anos de 1998 a 2003, o que rendeu 805 observações, informa que os bancos suavizaram resultados através das provisões para perdas, mecanismo igual ao identificado por Bath (1996) para esses bancos durante os anos de 1981 a 1991. Por sua vez, Sood (2012) ao analisar 878 bancos norte-americanos, entre os períodos de 2001 a 2009, constatou que os bancos suavizaram seus lucros em períodos de não recessão econômica (ho) e o mecanismo que utilizaram para tal foi a provisão para perdas; porém, quando a economia norte-americana entrou em recessão, esses bancos não tiveram estímulos para continuar suavizando seus lucros (h1).

Corroborando Sood (2012), Dolar (2016) de forma similar ao analisar os bancos norte-americanos entre os anos de 2007 a 2010, aumentou o número da amostra para 25.000 observações. Seus resultados sugeriram que os bancos, a fim de aumentar artificialmente seus lucros em declínio durante o período da crise financeira de 2008 , subestimaram suas provisões com perdas e que, de maneira oposta, durante o período de recuperação econômica, as superestimaram.

O trabalho de Bushman e Williams (2012) verificou 55.236 bancos de 27 países emergentes entre os anos de 1995 a 2006 e demonstrou que a variação do Produto Interno Bruto (PIB) desses países influenciaram de forma significativa a suavização de resultado cometida pelos bancos, por meio da provisão para perdas de crédito, sugerindo, assim, que a evolução do produto interno bruto influência na suavização de resultado.

No Brasil, Dantas et al. (2013) ao analisar o gerenciamento de resultado pelos bancos brasileiros, considerou a série trimestral de 207 bancos entre os anos de 2002 a 2010, em que também foi elencado como variável independente do modelo, a evolução trimestral do PIB brasileiro; os resultados desse estudo sugeriram que além da provisão para perdas sob crédito, os bancos brasileiros também utilizaram a mensuração a valor justo dos títulos de valores mobiliários para suavizarem seus resultados. 


\section{3- A relação do Brasil com outras economias mundiais e o fluxo de capital bancário}

Os dois principais parceiros econômicos do Brasil de acordo com BCB (2017a) é a China e os EUA. A relação existente do Brasil com os EUA demonstra que várias empresas brasileiras exportam seus produtos para essa economia e que de maneira mais específica, os bancos nacionais investem seus recursos no mercado norte-americano (BCB, 2017b).

Essa relação Brasil e EUA justificam os achados de uma pesquisa realizada anteriormente pelo BCB (2015) com os bancos que compõem o sistema financeiro nacional e exposto no Relatório de Economia Bancária e Crédito 2014. Foi constatado que os principais fatores de risco considerado pelo setor em 2014 e posteriormente; além da inadimplência, que não foi considerada por este estudo, 19\% dos bancos consultados apontaram o cenário externo e especificamente, a recuperação da economia dos EUA, como o principal fator de preocupação econômica da instituição, seguido da recessão econômica brasileira apontada por $10 \%$ dos bancos pesquisados. Os demais fatores apresentados foram a liquidez com também $10 \%$, inflação com $8 \%$, risco de mercado com $5 \%$, risco fiscal com $5 \%$, risco soberano com $4 \%$, falência com $4 \%$ e risco político com $5 \%$ (BCB, 2015).

Por causa disso, este estudo irá considerar como variáveis, apenas o cenário interno que é representado pela evolução trimestral do PIB do Brasil e o cenário externo considerado pela evolução trimestral do PIB dos EUA e de demais economias mundiais.

A relação Brasil e China por outro lado, possui como aspecto relevante a exportação de produtos brasileiros para o mercado interno Chinês. Essa relação é fruto de uma relação política e econômica crescente entre os governos desses dois países (Becard, 2011). Para Becard (2011), foi a partir da década de 1980 que Brasil e China intensificaram suas relações, o que o levou, em 2011, a um acordo bilateral entre essas duas economias. Nesse acordo ficou firmado, entre outras coisas, que a China emprestaria dinheiro para empresas brasileiras por meio do Banco da China no Brasil.

De acordo com o BCB (2017b), a China mantém empréstimos com empresas brasileiras que atuam nos setores de agricultura, pecuária, produção florestal, pesca, aquicultura, indústrias de transformação e em empresas de transportes e armazenagens. Esses setores da economia ao qual o Banco da China emprestou dinheiro já era previsível, pois de forma anterior, o Instituto de Pesquisa Econômica Aplicada (2011) já informava que a China tem interesse nas empresas brasileiras, desde que lhe ofereçam recursos básicos para fomentar sua economia doméstica, como é o caso da agricultura para sua população e de produtos semiacabados, da indústria de transformação.

Diante desses fatores, o BCB (2017a) demonstra por meio de seu Relatório de Estabilidade Financeira que a estabilidade econômica dos seus principais parceiros comerciais passa pela política econômica praticada via seus bancos centrais e que essas políticas podem favorecer ou desfavorecer o Brasil.

Uma terceira força econômica foi considerada também por este estudo, desta vez foi considerada a evolução do PIB dos sete países mais ricos do mundo (G7) como fator determinante para a suavização do resultado dos bancos brasileiros. O estudo de Resende e Amado (2007) foi o único estudo identificado que correlaciona fatores econômicos do Brasil com a evolução econômica do G7. Os autores demonstraram que existe uma relação oposta entre as economias da América Latina com as economias mais desenvolvidas, quando esta última se encontra em ascensão.

Resende e Amado (2007) ao considerar o período de crescimento das maiores economias mundiais identificou que as economias desenvolvidas apresentam entradas de capitais, enquanto as economias periféricas, como a brasileira e de outros países da América Latina, apresentam saídas de recursos. Para este estudo foi considerado que os principais agentes para essa migração de capital são os bancos, assim se esperou que diante da evolução do PIB das maiores economias mundiais os bancos brasileiros utilizassem seus recursos presentes para aplicar em economias externas.

Esta consideração ganhou relevância, pois de acordo com o BCB (2017), apenas os maiores bancos brasileiros são os que possuem investimentos, seja de pessoa física ou de pessoa jurídica no 
exterior. O G7 é composto pela Alemanha, Canadá, Estados Unidos, França, Itália, Japão e Reino Unido. A inclusão desse grupo se justifica pela sua importância econômica uma vez que representam a maior parte do PIB mundial.

\section{4- Índice de Eckel}

O pesquisador Norm Eckel (1981) propôs em seu artigo intitulado "the income smoothing hypothesis revised" um coeficiente de variação que tem como objetivo identificar se uma empresa suaviza de forma artificial os seus resultados ou não. Desde sua apresentação, este índice foi utilizado em diversos estudos, como por exemplo, Ashari, Koh, Tan e Wong (1994), Michelson, JordanWagner e Wootton (2000), Tseng e Wen Lai (2007), Ayoib e Nooriha (2009), Shubita (2016), que adotaram esse modelo para detectar suavização de renda em seus estudos.

O índice de suavização de Eckel leva em consideração a variação das vendas e a variação do lucro líquido. Dessa forma, o coeficiente de variação deve ser aplicado tanto nas vendas como no lucro líquido, conforme a equação abaixo:

Equação 1: Coeficiente de variação de Eckel

$$
C V=\frac{\sqrt{\sum(\Delta x i-\bar{x} \Delta)^{2}} / n-1}{\bar{x} \Delta}
$$

Onde, $d f=\left|C V_{\Delta \text { lucro líquido }} \div C V_{\Delta \text { vendas }}\right|$; assim:

$d f<1=$ A empresa suaviza artificialmente seus resultados;

$d f \geq 1$ = A empresa não suaviza artificialmente seus resultados.

No caso das instituições financeiras, as receitas decorrentes de intermediação financeira, prestação de serviços bancários e tarifas bancarias foram consideradas para determinar a variação das vendas; o lucro líquido se deu pela diferença entre o imposto de renda e a participação nos lucros por parte dos funcionários do resultado antes da tributação (BCB, 2017b).

O modelo tem como premissa os seguintes pontos: 1- As receitas com vendas não podem ser suavizadas de maneira artificial; 2- Há uma relação linear entre o lucro não suavizado e as receitas com vendas, de modo que quanto menor for essa relação mais as empresas suavizam seus lucros; 3Os custos fixos são constantes em determinado período de tempo, uma vez que eles não são alterados em função das vendas; 4- Os custos variáveis oscilam acompanhando a variação das vendas (Eckel, 1981).

Por fim, Albrecht e Richardson (1990) consideram os seguintes pontos como positivos para o modelo de Eckel: 1- Não é preciso utilizar nenhuma previsão de resultado; 2- Não há estudo multivariado dos custos, na verdade, todo custo é incluído no modelo uma vez que (Lucro = Receita Custo total); 3- Não há necessidade de um modelo comportamental dos gestores, pois o modelo considera que o comportamento de suavização é uma função do setor econômico; 4- Considera que a suavização de resultado causa impacto em períodos futuros, o que é capturado pelo desvio-padrão do coeficiente de variação.

\section{METODOLOGIA}

\section{1- Seleção da amostra}

A amostra inicial foi composta pelos 50 maiores bancos brasileiros, do seguimento b1- Banco comercial, múltiplo com carteira comercial ou caixa econômica, para os períodos de 2011 a 2017 divulgado pelo BCB e atualizada com os resultados financeiros de até o segundo trimestre de 2017. 
O critério de seleção para a amostra final se deu pelos bancos que permaneceram na lista para a série histórica de 2011 a 2017, totalizando 40 instituições bancárias, o que rendeu 26 trimestres e resultou em 1.040 observações.

\section{2- Modelos}

Foi aplicada a regressão logística binária com dados em painel. Seguindo a estrutura abaixo:

Equação 2: Modelo de regressão logit

$$
\operatorname{Pr}[y=1]=\frac{e^{\beta X i t+E i}}{1+e^{\beta X i t+E i}}
$$

O uso do modelo logístico binário se justifica pelo fato da variável dependente (y) ser qualitativa, em que o modelo tem como objetivo identificar a probabilidade de ocorrência de um determinado evento; $\beta$ é o coeficiente de cada variável, $X$ é a própria variável $i$ no momento $t$ (Greene, 2003). Para a implementação do modelo logit não foi considerado o aspecto quantitativo do índice de Eckel (1981), em que o coeficiente, $d f$, pode ser qualquer valor entre $0 \leq d f \leq+\infty$, mas se apegou ao aspecto qualitativo de quando $d f<1$ significa que a empresa suavizou seus resultados e $d f \geq 1$ representa que a empresa não praticou income smoothing. Assim, a variável dependente assume 1 quando o banco tiver suavizado seu resultado no período e 0 quando o banco não tiver suavizado.

O modelo do painel, para variáveis dependentes qualitativas, seguiu o exposto por Balgati (2005) e Hsiao (2014), em que argumentam que a utilização de dados em painel com efeitos fixos para modelos discretos, como o logit, não é recomendado. Balgati (2005) expõe que em um painel logit com efeitos fixos, devido à natureza discreta dos dados, o termo de erro não pode ser consistentemente estimado; essa dificuldade é conhecida como o problema dos parâmetros incidentais e mesmo utilizando transformações internas e simulação de Monte Carlo, o efeito fixo continuará enviesado. Balgati (2005) demonstra que o viés diminui à medida que mais tempo são considerados $(t)$, mas que ainda para um modelo com $i=1000$ e $t=20$ o viés será de $6,9 \%$. Uma vez que esse estudo utilizou i $=40$ e $t=26$, o mais viável é considerar painel com efeitos aleatórios.

A regressão teve como objetivo identificar se a prática de suavização ou não dos bancos brasileiros foram influenciadas pelo cenário econômico interno ou externo. O produto interno bruto considerado foi o que se encontra disponível na OECD (2017). O Ei representou o termo de erro do modelo. Assim, o $\beta$ Xit assumiu o exposto abaixo:

$$
\begin{aligned}
& \text { Equação 3: Regressão logit utilizada }
\end{aligned}
$$

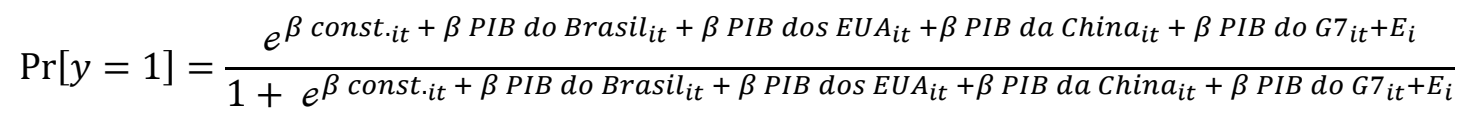

Fonte: Pesquisa (2018)

$\beta_{1}$ PIB do Brasil: Variação percentual do PIB real do Brasil para o período. $\beta_{2}$ PIB dos EUA: Variação percentual do PIB real dos EUA para o período.

$\beta_{3}$ PIB da China: Variação percentual do PIB real da China para o período.

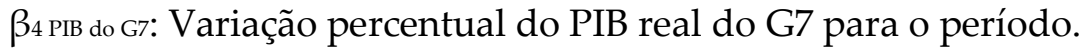


Em relação à variável dependente. $\mathrm{O} y=1$ indica que, em casos de suavização de resultado, do banco $i$ no período $t$ será considerado como 1. De forma oposta, caso o banco $i$ no período $t$ não tiver suavizado resultado, será atribuído 0 .

\section{ANÁLISE DOS RESULTADOS}

\section{1- Estatística descritiva}

Com exceção dos bancos Fator, que não suavizou seus resultados, todos os outros 39 bancos suavizaram seus lucros em pelo menos um dos períodos considerados, o que representa os 40 bancos da amostra.

Destes, 16 empresas demonstraram suavizar seus resultados pelo menos na metade do tempo considerado. Estas empresas apresentam moda igual a 1 e frequências que variam de 50,00\% a 80,77\%. Os bancos que possuem moda igual a 1 foram a Socopa, Alfa, JP Morgan Chase, Banrisul, BRB, Industrial do Brasil, Bco Daycoval S.A, Caixa Econômica Federal, Bradesco, BMG, HSBC, Bco Modal, John Deere, Deutsche, Rendimento, Máxima.

As outras 23 instituições bancárias suavizaram seus resultados pelo menos em algum período. Essas empresas possuem moda igual a 0, e frequências que variam de 3,85\% a 42,31\%. São elas o BNP Paribas, BBM, Sofisa, Societe Generale, Pine, VR, Banif, Mercantil do Brasil, Banestes, Citibank, Credit Suisse, Indusval, Votorantim, CreditAgricole, Morgan Stanley, BB, Santander, Itaú, Safra, ABC Brasil, ING, Bancoob, Intermedium.

Tabela 1: Estatística descritiva

\begin{tabular}{|c|c|c|c|}
\hline Bancos & QTD & Frequência & Moda \\
\hline BNP PARIBAS & 1 & $3.85 \%$ & 0 \\
\hline BBM & 2 & $7.69 \%$ & 0 \\
\hline SOFISA & 2 & $7.69 \%$ & 0 \\
\hline SOCIETE GENERALE & 3 & $11.54 \%$ & 0 \\
\hline PINE & 3 & $11.54 \%$ & 0 \\
\hline VR & 3 & $11.54 \%$ & 0 \\
\hline BANIF & 4 & $15.38 \%$ & 0 \\
\hline MERCANTIL DO BRASIL & 7 & $26.92 \%$ & 0 \\
\hline BANESTES & 7 & $26.92 \%$ & 0 \\
\hline CITIBANK & 8 & $30.77 \%$ & 0 \\
\hline CREDIT SUISSE & 8 & $30.77 \%$ & 0 \\
\hline INDUSVAL & 8 & $30.77 \%$ & 0 \\
\hline VOTORANTIM & 8 & $30.77 \%$ & 0 \\
\hline CREDIT AGRICOLE & 8 & $30.77 \%$ & 0 \\
\hline MORGAN STANLEY & 8 & $30.77 \%$ & 0 \\
\hline BB & 9 & $34.62 \%$ & 0 \\
\hline SANTANDER & 9 & $34.62 \%$ & 0 \\
\hline ITAU & 10 & $38.46 \%$ & 0 \\
\hline SAFRA & 10 & $38.46 \%$ & 0 \\
\hline ABC-BRASIL & 10 & $38.46 \%$ & 0 \\
\hline ING & 11 & $42.31 \%$ & 0 \\
\hline BANCOOB & 11 & $42.31 \%$ & 0 \\
\hline INTERMEDIUM & 11 & $42.31 \%$ & 0 \\
\hline SOCOPA & 13 & $50.00 \%$ & 1 \\
\hline
\end{tabular}




\begin{tabular}{llll}
\hline ALFA & 13 & $50.00 \%$ & 1 \\
JP MORGAN CHASE & 14 & $53.85 \%$ & 1 \\
BANRISUL & 14 & $53.85 \%$ & 1 \\
BRB & 14 & $53.85 \%$ & 1 \\
INDUSTRIAL DO BRASIL & 14 & $53.85 \%$ & 1 \\
BCO DAYCOVAL S.A & 15 & $57.69 \%$ & 1 \\
CAIXA ECONOMICA FEDERAL & 16 & $61.54 \%$ & 1 \\
BRADESCO & 16 & $61.54 \%$ & 1 \\
BMG & 16 & $61.54 \%$ & 1 \\
HSBC & 16 & $61.54 \%$ & 1 \\
BCO MODAL S.A. & 16 & $61.54 \%$ & 1 \\
JOHN DEERE & 17 & $65.38 \%$ & 1 \\
DEUTSCHE & 18 & $69.23 \%$ & 1 \\
RENDIMENTO & 19 & $73.08 \%$ & 1 \\
MÁXIMA & 21 & $80.77 \%$ & 1 \\
\hline
\end{tabular}

${ }^{1}$ QTD. - Quantidade de vezes que a empresa suavizou resultado. Fonte: Pesquisa (2018).

Uma análise complementar foi o da ocorrência de income smoothing ao longo do período para a amostra; essa análise teve por objetivo identificar a utilização desse mecanismo pelo setor ao longo do tempo. Foi visto que em 2011, o número de empresas que suavizaram resultados em pelo menos algum período, foi de 35 instituições. Em 2012 esse número diminuiu para 32 instituições bancárias ou $80 \%$. Já em 2013 , apenas 28 empresas, que corresponde a $70 \%$ da amostra.

Em 2014 houve modesto crescimento no número de empresas que passou para 29 instituições, 72,50\%. Em 2015, foram 32 instituições bancárias que suavizaram seus lucros o que representa $80 \%$ das empresas. No ano de 2016, foram 30 bancos que suavizaram resultado o que corresponde a $75,00 \%$ da amostra; e até o primeiro semestre de 2017, o número de instituições bancárias da amostra que suavizaram resultado foi de 21 ou $52,50 \%$.

Foi efetuada uma análise com o objetivo de identificar entre o ano de 2011 até o segundo trimestre de 2017 em quais trimestres, em média, a prática de income smoothing era mais utilizada. Foi achado que, ao longo desses 6 anos e 6 meses, a média das empresas que suavizaram resultado no primeiro trimestre foi de aproximadamente $36 \%$; no segundo trimestre de $44 \%$; no terceiro trimestre de $36 \%$ e no quarto trimestre de $43 \%$. Dessa forma, para o período analisado, as maiores práticas de suavização de resultado ocorreram no segundo e quarto trimestre de cada ano.

Os resultados da estatística descritiva demonstraram que $60 \%$ da amostra não suavizou seus resultados na maior parte do período considerado neste estudo. Este resultado, de forma preliminar, corrobora para o exposto por Sood (2012) de que bancos possuem pouco estímulos a suavização de resultado, quando sua economia interna passa por um momento de declínio.

O fato dos bancos suavizarem mais seus resultados durante o segundo e quarto trimestre de cada ano pode ter suas raízes na Teoria da Legitimidade, como exposto por Côté (2016), onde os bancos ao final de cada semestre ajustaria seu lucro a um nível que fosse aceitável pela própria instituição, no sentido de fazer caixa e pelos investidores e demais stakeholders que possuem também interesse nos resultados financeiros apresentados.

\section{2- Resultado do cenário econômico interno e externo}

Ao analisar a prática de suavização de resultado em função do desenvolvimento do cenário econômico interno e externo, dado pela evolução trimestral do PIB de cada variável, foi encontrado 
que o income smoothing foi influenciado de forma positiva pelo PIB dos EUA, e de forma negativa pelo PIB do G7.

A evolução do PIB dos EUA apresentou p-value significativo a 1\%, o coeficiente de 0,793442 é positivo e demonstra que o cenário interno norte-americano influenciou positivamente a suavização de resultado dos bancos, e o VIF de 2,3, informou que a variável não tem problema de multicolinearidade. A razão de chances apresentada pelo PIB dos EUA é de 2,21, o que significa que um banco brasileiro terá aproximadamente duas vezes mais chances de suavizar seus resultados, em resposta ao crescimento de um ponto percentual do PIB dos EUA.

Em relação ao G7, o p-value apresentado foi significativo a 1\%. O VIF de 0,61 descartou problemas de multicolinearidade. O coeficiente de $-1,36773$ demonstrou que a evolução do PIB dessas 7 economias mundiais não ofereceu estímulos a suavização de resultado dos bancos brasileiros. A razão de chances de que o cenário econômico do G7 viesse determinar a prática de suavização de resultado foi de apenas 1,2 vezes, o que implica dizer que as chances do G7 determinarem a não suavização dos bancos brasileiros, devido ao seu crescimento econômico ter sido de 1,2 vezes.

Uma vez que os EUA e o G7 se apresentaram significativos e divergentes entre si, uma razão de chances maior para os EUA demonstrou que os bancos foram probabilisticamente mais sensíveis a variação da economia Norte-Americana no que diz respeito a prática de income smoothing. Esse resultado pôde ser explicado pelo fato dos EUA possuírem o maior mercado financeiro do mundo e sua política interna ter sido pró investidora, enquanto vários países do G7 apesar de possuírem mercados financeiros desenvolvidos serem pró stakeholders como Alemanha, Itália e Japão.

Em relação ao modelo, o mesmo possuiu uma capacidade de predizer corretamente $60,10 \%$ dos casos. A especificidade do modelo é de $96,65 \%$, enquanto sua sensibilidade foi de 4,60\%. Esses resultados demonstraram que o modelo possuiu uma baixa sensibilidade ao tentar prever se um banco suavizou seu resultado, ao mesmo tempo em que buscou especificar quais bancos não suavizariam seus resultados tendo em face o cenário econômico. No entanto, uma vez que o objetivo deste estudo não foi realizar previsão, mas determinar quais cenários econômicos influenciaram na suavização de resultado dos principais bancos do Brasil, esses resultados foram satisfatórios.

Tabela 2: Resultado da regressão

\begin{tabular}{|c|c|c|c|c|c|}
\hline Eckel & Coeficiente & & Razão de Chances & Erro Padrão & $\begin{array}{c}\text { Fator de Inflação da } \\
\text { variância }\end{array}$ \\
\hline PIB dos EUA & 0.793442 & $* * *$ & 2.210994 & 0.261402 & 2.30 \\
\hline PIB do Brasil & 0.046374 & & 1.047466 & 0.075712 & 2.21 \\
\hline PIB do G7 & -1.367730 & $* * *$ & 0.254686 & 0.485200 & 1.20 \\
\hline PIB da China & -0.388730 & & 0.677918 & 0.277768 & 1.18 \\
\hline Constante & 0.328242 & & 1.388525 & 0.566317 & - \\
\hline
\end{tabular}

Número de casos corretamente previstos $=625(60,1 \%)$

Previsto 


\begin{tabular}{|c|c|c|c|}
\hline Suaviza & 394 & 19 & 413 \\
\hline & 1000 & 40 & 1040 \\
\hline Especificidades & $96.65 \%$ & Sensibilidade & $4.60 \%$ \\
\hline
\end{tabular}

O fato do cenário econômico interno não ter sido significativo para determinar a prática de income smoothing, corroborou os indícios levantados por Sood (2012) e Dolar (2016), de que cenários econômicos internos recessivos não ofereceram estímulos para a suavização de resultado. Em especial, os resultados encontrados ao analisar o $p$-value do PIB do Brasil demonstraram que os bancos brasileiros foram indiferentes ao cenário econômico interno, ou seja, o cenário econômico do Brasil de forma geral não influenciou os bancos brasileiros a suavizarem seus resultados. Além disso, o PIB do Brasil apresentou uma razão de chances quase linear com a prática de income smoothing.

Uma vez que a prática de suavização de resultado foi significativa com a evolução do PIB dos EUA e que os agentes do mercado demonstraram preocupação com o cenário econômico NorteAmericano, segundo o BCB (2015), a suavização de resultado feita pelos maiores bancos do Brasil, segundo os resultados desta pesquisa, não foi motivada por expectativas externas ou pelo declínio da economia brasileira para o período analisado, mas foi motivado pelas preocupações com a economia dos EUA e a forma como esta poderia influenciar taxas de juros, fluxo de capital, retorno de investimentos, entre outros fatores.

Cabe a outras pesquisas futuras buscar determinar o motivo pelo qual o cenário econômico interno não ter sido estatisticamente significativo; se isso se deveu ao fato de apenas seis bancos comerciais dominarem a maior parte do mercado brasileiro, para o período analisado, ou se o fato da maior parte dos bancos comerciais não ser listados na bolsa de valores brasileiras fez que eles fossem indiferentes a prática de suavização uma vez que não possuem necessidade de atender aos investidores locais. Assim, o motivo pelo qual um banco de menor expressão no mercado poderia gerir seus resultados seria motivado por algum interesse específico (Kanagaretnam, Lobo \& Yang, 2004).

\section{CONSIDERAÇÕES FINAIS}

Este artigo teve como objetivo analisar se o cenário econômico, interno ou externo, influenciou na prática de income smoothing, realizados pelos principais bancos comerciais, múltiplos com carteiras comerciais e caixas econômicas do Brasil, entre o primeiro trimestre de 2011 e o segundo trimestre de 2017.

O estudo se baseou nos trabalhos de Galai e Winer (2003), Liu e Ryan (2006), Sood (2012) e Dolar (2016) que sugeriram que os bancos não tendem a suavizar resultados quando o cenário econômico interno foi recessivo. E nos de DeFound e Park (1997), Healy e Walen (1999), Gao e Zhang (2015) evidenciaram que a prática income smoothing foi realizada com base nas expectativas sobre cenários econômicos futuros.

Os resultados das análises empíricas forneceram evidências de que a maioria dos bancos considerados por este estudo praticaram income smoothing ao menos em um período apesar do momento recessivo vivido pela economia brasileira ao longo de 2015 e 2016. Porém, que não foi prática dominante da maior parte da amostra suavizar seus lucros na maior parte do período considerado, o que levantou evidências que, de fato, os bancos não tendem a suavizar seus resultados quando o cenário econômico foi desfavorável (Sood, 2012; Dolar, 2016). Ainda foi visto que a maior parte das suavizações de resultado ocorreram no segundo e quarto trimestre de cada ano. 
A principal conclusão chegada por este estudo foi que os bancos da amostra não levaram em consideração o cenário econômico interno, antes, praticaram income smoothing por algum outro motivo. Além do fato, de que a evolução do PIB dos EUA ofereceu estímulos para a prática se suavização de resultado. Nesse contexto os bancos suavizaram seus resultados em resposta ao cenário presente atravessado pelo EUA, que era de recuperação e volta de crescimento após a crise financeira de 2008.

Por fim, esse tema merece um maior aprofundamento, pois de acordo com Jayaraman (2008) as empresas listadas em bolsa podem suavizar seus resultados com o objetivo de passar alguma sensação aos investidores, governo ou a sociedade no geral.

\section{REFERÊNCIAS}

Ahmed, A., Takeda, C., \& Thomas, S. (1999). Bank loan loss provisions: a reexamination of capital management, earnings management and signaling effects. Journal of Accounting and Economics, vol. $28, \mathrm{n}^{\mathrm{o}} 1$.

Albrecht, D., \& Richardson, F. (1990). Income smoothing by economy sector. Journal of Business Finance $\mathcal{E}$ Accountint, vol. 17, no 5.

Ashari, N., Koh, H. C., Tan, S. L., \& Wong, W. H. (1994). Factors affecting income smoothing among listed companies in Singapore. Accounting and Business Research, Vol. 24, No. 96, pp. 291-301.

Ayoib, C. A., \& Nooriha, M. (2009). Board independence, ownership structure, audit quality and income smoothing activities: a study on Malaysian Market. Journal of Modern Accounting and Auditing, Vol. 5, No. 11, pp. 1-13.

Balgati, B. H. (2005). Econometric analysis of panel data. Third Edition, Wiley \& Sons.

Banco Central do Brasil (2015). 50 Maiores Bancos e Consolidado do Sistema Financeiro Nacional. Endereço eletrônico < http://www4.bcb.gov.br/top50/port/top50.asp>. Acesso em 10 de junho de 2016.

. Dados Selecionados de Entidades Supervisionadas - IF. Data. Endereço eletrônico < https://www3.bcb.gov.br/informes/relatorios?lingua=pt> Acesso em 10 de junho de 2016.

Banco Central do Brasil (2017a). Relatorio de estabilidade financeira. Endereço eletrônico < http://www.bcb.gov.br/htms/estabilidade/2017_10/refPub.pdf> Acesso em 17 de março de 2018.

Banco Central do Brasil (2017b). dados das instituições financeiras. Endereço eletrônico < https://www3.bcb.gov.br/ifdata/> Acesso em 17 de março de 2018.

Bath, V. N. (1996). Bank and income smoothing: an empirical analysis. Applied financial economics, vol. 6 , no 6, pp. 505-510.

Becard, S. R. (2011). O que esperar das relações brasil-china? revista de sociologia e política v. 19, n⿳o suplementar: 31-44 nov. 2011. 
Beidleman, C. R. (1973). Income smoothing: the role of management. The accounting review, vol. 48, $\mathrm{n}^{\circ}$ 4, pp. 653-667.

Blanchard, O. (2007). Macroeconomia. $4^{a}$ edição, Prentice Hall.

Bushman, R. M., \& Williams, C. D. (2012). Accounting discretion, loan loss provisioning, and discipline of Banks' risk-taking. Journal of Accounting and Economics, vol. 54, no 1 .

Cahan, S. F., Liu, G., \& Sun, J. (2008). Investor protection, income smoothing, and earnings informativeness. Journal of international accounting research. Vol. 7, no 1.

Chaney, P. K., Lewis, C. M. (1995). Earnings management and firm valuation under asymmetric information. Journal Corporate Finance. vol. 1, nํ- 3-4, pp. 319-345.

Copeland, R. M. (1968). Income smoothing. Journal of accounting research, vol. 6, pp. 101-116.

Côté, G. B. (2016). Profit and the legitimacy of the Canadian banking industry. Accounting, auditing E accountability jornal, vol. 19, n⿳o 4 .

Dantas, J. A., Medeiros, O. R., Galdi, F. C., \& Costa, F M. (2013). Gerenciamento de Resultados em Bancos com Uso de TVM: Validação de Modelo de Dois Estágios. Revista de Contabilidade e Finanças. - USP, São Paulo, v. 24, nº 61, pp. 37-54.

DeFound, M., \& Park, C. W. (1997). Income smoothing in antecipation of future earnings. Journal of accounting and economics, vol. 23, no 2, pp. 115-139.

Dolar, B. (2016). Income smoothing pratices of US banks around the 2008 financial crisis. The international journal of business and finance research, vol. 10, $\mathrm{n}^{\mathrm{o}} 1$.

Eckel, N. (1981). The income smoothing hypothesis revisited. Abacus, vol. 17 no 17.

Galai, E. S., \& Winer, Z. (2003). Accounting values versus market values and earnings management in banks. Working paper, The Hebrew University of Jerusalem, Israel.

Gao, L., \& Zhang, J. H. (2015). Firms' earnings smoothing, corporate social responsibility, and valuation. Journal of corporate finance, vol. 32, nํㅡ, C. $108-127$.

Goulart, M. C. (2007). Gerenciamento de dados contábeis em instituições financeiras no Brasil. Tese de doutorado em ciências contábeis. Universidade de São Paulo.

Greene, W. H. (2003). Econometric analysis. New Jersey, Pretice Hall, 5oe edição.

Graham, J. R., Harvey, C. R., \& Rajgopal, S. (2005). The economic implications of corporate financial reporting. Journal Accounting Economics. v. 40, dezembro, pp. 3-73.

Gulati, R., Nohria, N., \& Wohlgezogen, F. (2010). Roaring out of recession. Harvard Business Review, vol. 88, março 2010. 
Healy, P. M., \& Wahlen, J. M. (1999) A Review of the Earnings Management Literature and Its Implications for Standard Setting. Accounting Horizons, v. 13, n. 4, pp. 365-383.

Hsiao, C. (2014). Analysis of Panel Data. Third Edition. Cambridge University Press.

Instituto de Pesquisa Economica Aplicada. (2011). As relações bilaterais Brasil - China: A ascensão da China no sistema mundial e os desafios para o Brasil. Comunicados do IPEA no 85 .

Jayaraman, S., (2008). Earnings volatility, cash flow volatility, and informed trading. Journal Accounting Research. v. 46, nº 4, pp. 809-851.

Kanagaretnam, K., Lobo, G. J., \& Yang, D. H. (2004). Joint tests of signaling and income smoothing through bank loan loss provisions. Contemporary accounting research, v. 21, no 4 .

Kilic, E., Lobo, G. J., Ranasinghe, T., \& Sivaramakrishnan K. (2013). The impact of SFAS 133 on income smoothing by banks through loan loss provisions. The accounting review. v. $88, \mathrm{n}^{\mathrm{o}} 1$.

Lambert, R. A. (1984). Income smoothing as rational equilibrium behavior. The accounting review, vol. 59 , no 4 , pp. 604-618.

Liu, C. C., \& Ryan, S. G. (2006). Income Smoothing over the Business Cycle: Changes in Banks' Coordinated Management of Provisions for Loan Losses and Loan Charge-Offs from the Pre-1990 Bust to the 1990s Boom. The accounting review, vol. 81, no 2 .

Michelson, S. E., Jordan-Wagner, J., \& Wootton, C. W. (2000). The relationship between the smoothing of reported income and risk-adjusted returns, Journal of Economics and Finance, Vol. 24, $\mathrm{n}^{\mathrm{o}}$ 2, pp. 141-159.

Nason, R. S., \& Patel, P. C. (2016). Is cash king? Market performance and cash during a recession. Journal of Business Research, vol.69, nº 10, pp. 4242-4248.

Organisation For Economic Co-Operation and Development. (2017) Quartely National Accounts: Quartely Growth Rates of real GDP, change over previous quarter. Endereço eletrônico < https://stats.oecd.org/index.aspx?queryid=350> Acesso em: 10 de novembro de 2017.

Resende, F. C., \& Amado, A. M. (2007). Liquidez internacional e ciclo reflexo: algumas observações para a América Latina. Revista de Economia Política, v. 27, no 1 (105), pp. 41-59, janeiromarço/2007.

Richardson, S. (2006). Over-investment of free cash flow. Review of Accounting Studies, v. 11, no 2-3, pp. 159-189.

Shubita, M. F. (2015). The impact of income smoothing on earnings quality in emerging markets: Evidence from GCC markets. Journal of accounting in emerging economies, v. 5, noㅡ 3, pp. 299-324.

Sood, H. A. (2012). Loan loss provisioning and income smoothing in US banks pre and post the financial crisis. International Review of Financial Analysis, v. 25, dezembro 2012, pp. 64-72. 
Tseng, L. J., \& Wen, L. C. (2007). The relationship between income smoothing and company profitability: an empirical study. International Journal of Management, v. 24, no 4, pp. 727-733.

Tucker, J. W., \& Zarowin P. A. (2006). Does income smoothing improve earnings informativeness? The Accounting Review, v. 81, no 1 . 